\title{
Tragische Dispositionen der Moderne Stimmung UND AURA IM WANDEL DES LANDSCHAFTSBEGRIFFS
}

Dirk Michael Hennrich ${ }^{1}$

(Investigador do Centro de Filosofia da Universidade de Lisboa)

Möchte man einen Eindruck davon gewinnen, welche Bedeutung das Stimmungsvolle, die Stimmungen und die Stimmung im Verlauf des 19. Jahrhunderts erhalten haben, wie die Stimmung langsam aber stetig umbrach, sich tragisch ergossen hat und wie sie zugleich mit der Landschafstbetrachtung und der Landschaftsmalerei in Verbindung steht, müssen der Begriff der Stimmung bei Alois Riegl und Georg Simmel und der Begriff der Aura bei Walter Benjamin hervorgehoben werden.

Ohne eine große Neuigkeit zu verkünden, ist zunächst davon auszugehen, dass Epochenwechsel grundlegend Stimmungswechsel sind und dass nicht nur irgendwelche technischen Erfindungen eine Epoche beenden und die Menschheit über eine weitere Schwelle tragen, sondern dass die neuen Sichtweisen und die daraus resultierenden kulturellen Innovationen von einem allgemeinen Unbehagen ausgelöst oder zumindest eingelöst werden. Das Unbehagen der Kultur um die letzte Jahrhundertwende, von dem Freud sprach, und das seiner Ansicht nach aus einem gestörten Sexualhaushalt hervorbrach, ist nicht einfach das Unbehagen der Moderne, die sich von einem allgemeinen Wohlsein der vorherigen Epochen unterscheidet. Alle Epochen haben ihr Unbehagen, ohne das die ihr folgenden Epochen nicht denkbar wären und ohne das es nie zu einer Befreiung aus den eingefrorenen Normen der Vorherigen kommen würde. Der Stimmungs-

${ }_{1}$ Dirk.Hennrich@gmx.ch

Philosophica, 46, Lisboa, 2015, pp. 19-30. 
wechsel ist somit mehr als nur ein Paradigmenwechsel, er ist der eigentliche Klimawandel einer Kulturepoche und beschreibbar mit der schweren zitternden Atmosphäre eines Sommernachmittags, an dem alles still und alles erstarrt zu sein scheint und sich in dieser Erstarrung zugleich das kommende Gewitter ankündigt. Diesem Stimmungswechsel nachzugehen und ihn auf seinen Gehalt zu prüfen ist somit, wenn auch nur ansatzweise, das hier zu bewerkstelligende Unternehmen.

Der Stimmungswechsel, ist ein zentraler Punkt der antiken Tragödientheorie und auch im Trauerspiel, die beide nicht nur an dem schicksalhaften (Unter-)Gang der Helden zu erkennen sind, sondern eben durch den Moment des plötzlichen Umschlags, der Peripetie, durch den Augenblick der Katastrophe, ihren tragischen Gehalt offenlegen. Der Stimmungswechsel ist das ausschlaggebende Element der Tragödie und ist begründet im Wechsel der Töne, so etwa bei Hölderlin, der an der Epochenschwelle um 1800 die Stimmung und den Stimmungswechsel in seine Tragödientheorie unausgesprochen mit einbezieht.

Was aber geschieht im 19. Jahrhundert, das die Tragödie als einen Gegenstand der Philosophie neu entdeckt und welche Verbindung besteht zwischen dieser Neigung und der grundlegenden Veränderung im Landschaftsempfinden am Beginn der romantischen Landschaftsauffassung und der romantischen Landschaftsmalerei als der Anfang vom Ende derselben?

Um 1800 lassen „die schmerzlich erfahrene Krise der klassischen Kunst und das Ungenügen an der bloßen Naturstudie einen neuen romantischen Landschaftsbegriff hervortreiben" ${ }^{2}$, wobei Carl Ludwig Fernow (1763-1808) und Carl Gustav Carus (1789-1869) zu den ersten gehören, die diesen Wechsel theoretisch einleiten und festhalten und dabei bereits die Grundthesen von Georg Simmels Philosophie der Landschaft andeuten. In Simmels Aufsatz, der 1913 erscheint und als Grundlagentext einer neuen philosophischen Disziplin betrachtet werden muss, ist die Stimmung ,ein wesentliches oder vielleicht das wesentliche Moment [...] das die Teilstücke [einer angeschauten Vielzahl natürlicher Erscheinungen] zu der Landschaft als einer empfundenen Einheit zusammenbringt $[\ldots]^{\text {‘3 }}$.

Die Landschaftsmalerei erhält um 1800 immer deutlicher die Aufgabe Stimmungen hervorzurufen und so schreibt bereits Fernow im Vorgriff auf die Ausführungen von Simmel:

Die Landschaftsmalerei [...] bedarf keines bestimmten Inhalts. In ihr ist blos ein Mannigfaltiges landschaftlicher Gegenstände, gemäs einer Idee

\footnotetext{
2 Werner Busch (Org.), Landschaftsmalerei, Berlin: Dietrich Reimer Verlag, 1997, p. 242.

3 Georg Simmel, „Philosophie der Landschaft“, Gesamtausgabe (GSG) Band 5, p. 479.
} 
zu einem Ganzen verbunden, hinreichend durch seinen Gesamteindruk eine gewisse Stimmung zu bewirken [.] Der einzelne Gegenstand hat für sich keine ästhetische Bedeutung und kein Interesse. [...] Die ästhetische Stimmung, in die Natur und Kunst das Gemüth versetzen, besteht eigentlich darin, dass das Gemüth sich von aller Bestimmung frei, und doch beschäftigt fühlt. ${ }^{4}$

Auch Carl Gustav Carus schreibt in seinen dritten Brief der Briefe über die Landschaftsmalerei deutlich über die Stimmungen der Landschaften und hat dabei ohne Zweifel auch die Landschaftsbilder seines Freundes Caspar David Friedrich im Sinn, der wiederum ein treffendes und bekanntes Beispiel für die aufkommende Hervorhebung des Stimmungsvollen ist. Für Carus ist die „Hauptaufgabe landschaftlicher Kunst [die] Darstellung einer gewissen Stimmung des Gemüthlebens (Sinn) durch die Nachbildung einer entsprechenden Stimmung des Naturlebens (Wahrheit). ${ }^{\text {“5 Die Bindung des }}$ Begriffs der Stimmung an die sentimentalistische Landschaftsbetrachtung lässt sich durch das ganze 19. Jahrhundert verfolgen und findet sich theoretisch reflektiert am Umbruch zur Moderne in Autoren wie Alois Riegl und eben Georg Simmel wieder. Bei Riegl zeigt sich nun auch eine Bestimmung der Landschaftsmalerei und insbesondere der Stimmung, die weitreichende Konsequenzen für die Definition der Aura bei Walter Benjamin haben wird.

In seinem Beitrag Stimmung als Inhalt moderner Kunst von 1899 schreibt Riegl über seine Erfahrung des Stimmungsvollen an einem Frühsommernachmittag in einer Alpenregion, einer Landschaft, deren Stimmung ihn von aller Enge und Bedrängnis befreit und aus die er doch durch die plötzliche Bewegung eines aufspringenden Tieres aufgeschreckt wird. Hier, in dieser stimmungsvollen Landschaft, aber auch in anderen Landschaften, ahnt der Betrachter, so schreibt Riegl,

dass weit über den Gegensätzen, die ihm seine unvollkommenen Sinne in der Nähe vortäuschen, ein Unfassbares ${ }^{6}$, eine Weltseele [vorhanden ist, die] alle Dinge durchzieht und sie zu vollkommenem Einklange vereinigt. Diese Ahnung aber der Ordnung und der Gesetzlichkeit über dem

${ }^{4}$ Carl Ludwig Fernow, Römische Studie, 2. Theil, Zürich, 1806, p. VI-X, 21-25, 36, 100, 110f. In Werner Busch Werner, op. cit., p. 246.

5 Carl Gustav Carus, Neun Briefe über Landschaftsmalerei geschrieben in den Jahren 1815-1824, Leipzig, 1831, p. 41.

6 Cf. Philipp Otto Runge, „Text zur Kunstausstellung in Weimar“, 1802, Hinterlassene Schriften Bd. 1, Hamburg 1840, pp. 5-7. in Werner Busch, op. cit., p. 242. „[E]s drängt sich alles zur Landschaft, sucht etwas bestimmtes in dieser Unbestimmtheit und weiß nicht, wie es anzufangen?“" 
Chaos, der Harmonie über den Dissonanzen, der Ruhe über den Bewegungen nennen wir die Stimmung. Ihre Elemente sind Ruhe und Fernsicht. ${ }^{7}$

Walter Benjamin definiert den Begriff der Aura in der Abhandlung Das Kunstwerk im Zeitalter seiner technischen Reproduzierbarkeit von 1936 und in seinem Essay Kleine Geschichte der Photographie von 1931 nun nahezu identisch, doch für die Herkunft seiner Auffassung der Aura aus der Landschaftsbetrachtung, ist der Kunstwerkaufsatz von tragender Bedeutung. Hier schreibt Benjamin:

Es empfiehlt sich, den oben für geschichtliche Gegenstände vorgeschlagenen Begriff der Aura an dem Begriff einer Aura von natürlichen Gegenständen zu illustrieren. Diese letztere definieren wird als einmalige Erscheinung einer Ferne, so nah sie sein mag. An einem Sommernachmittag ruhend einem Gebirgszug am Horizont oder einem Zweig folgen, der seinen Schatten auf den Ruhenden wirft - das heißt die Aura dieser Berge, dieses Zweiges atmen. An der Hand dieser Beschreibung ist es ein Leichtes die gesellschaftliche Bedingtheit des gegenwärtigen Verfalls der Aura einzusehen. Er beruht auf zwei Umständen die beide mit der zunehmenden Bedeutung der Massen im heutigen Leben zusammenhängen. Nämlich: Die Dinge sich räumlich und menschlich ,näherzubringen “ist ein genau so leidenschaftliches Anliegen der gegenwärtigen Massen, wie es ihre Tendenz einer Überwindung des Einmaligen jeder Gegebenheit durch die Aufnahme von deren Reproduktion ist. Tagtäglich macht sich unabweisbarer das Bedürfnis geltend, des Gegenstands aus nächster Nähe im Bild, vielmehr im Abbild, in der Reproduktion, habhaft zu werden. ${ }^{8}$

Was somit bei einem direkten Vergleich der Beschreibung der Aura bei Benjamin mit den Ausführungen von Riegl hervorspringt, ist die Nachbarschaft zu Riegl's Deutung der Stimmung, da sie ebenfalls durch den Sommernachmittag, die Gebirgsregion, die Ferne, den Ruhenden und die Ruhe an sich als Grundlage für die Stimmungsbestimmung aufgebracht wird. Doch der heiße Sommernachmittag, von dem Riegl spricht, der zu Beginn von Simmel's Aufsatz Böcklins Landschaften von 1895 stimmungsvoll auftaucht und der bei Benjamin den Begriff der Aura aus seiner „natürlichen“ Erscheinung erklärt, ist auch eine Metapher für die Zeit des

\footnotetext{
7 Alois Riegl, „Stimmung als Inhalt moderner Kunst“, in Die Graphischen Künste, pp. 47-56, Wien, Jahrgang XXII, 1899, Heft I, p. 48.

8 Walter Benjamin, Das Kunstwerk im Zeitalter seiner technischen Reproduzierbarkeit, Edition Suhrkamp, 1963, p. 15. Cf. Josef Fürnkäs, Aura, in Benjamin Begriffe, 1. Band, pp. 95-145, Frankfurt a. M.: Suhrkamp Verlag, 2000.
} 
Umbruchs, für die starre, zitternde Atmosphäre einer Epoche, die sich ihrem Ende entgegen neigt.

Es ist nun zu bemerken, dass diese Verfallsepoche, so wie Benjamin sie nennt, zugleich die Epoche des Landschaftsbegriffs ist, wie er aus der Malerei und im Zuge der Erfindung und Entwicklungsgeschichte der Fotografie, in die Philosophie eindringt und die in ihm eingelagerten Begriffe der Stimmung und eben den der Aura mit sich führt. Das 19. Jahrhundert, an dem Benjamin seine eigene Kunst- und Geschichtstheorie erarbeitet, ist das Zeitalter der Dekadenz, Paris ist die Hauptstadt dieser Epoche und Baudelaire ist ihr Dichter. In Benjamins Passagen-Werk ist sie beschrieben durch ihren ,narkotischen Historismus [, ihre] Maskensucht" durch ihre Langeweile ${ }^{I 0}$ und der Idee der ewigen Wiederkunft, deren Zeitgeist Benjamin erkennt, wenn er den Gedanken Zarathustras und „die gestickte Devise des Schlummerkissens ,Nur ein Viertelstündchen“"11 miteinander in Verbindung bringt. Es ist eine Epoche unter dem schwülen, schwermütigen und träumerischen Druck einer zerfallenden Aura, denn das „Leben im Bannkreis der ewigen Wiederkehr gewährt eine Existenz, die aus dem Auratischen nicht heraustritt." ${ }^{12}$ Sie ist die „Verfallsepoche“"13, zu der Nietzsche zählt und die Nietzsche in seiner Zeitkritik unablässig als dekadent bezeichnet, die seiner Ansicht nach in Richard Wagner ihren höchsten Ausdruck fand und die von Thomas Mann in seinem Roman Buddenbrooks durch den „Verfall einer Familie“ beschrieben wurde. Das Unbehagen der Kultur, das Tragische der Moderne, ist im Verenden der Stimmung, der Aura, aufzufinden, dieser letzte Ausdruck der Andacht, wie

9 Walter Benjamin, Das Passagen-Werk, Frankfurt a. M.: Suhrkamp Verlag, 1991, p. 493; K 1a, 6.

${ }^{10} \mathrm{Ibid}$. p. 161, D 2, 7, „Langeweile haben wir, wenn wir nicht wissen, worauf wir warten. $\mathrm{Da} ß$ wir es wissen oder zu wissen glauben, das ist fast immer nichts als der Ausdruck unserer Seichtheit oder Zerfahrenheit. Die Langeweile ist die Schwelle zu großen Taten. - Nun wäre zu wissen wichtig: der dialektische Gegensatz zur Langeweile?“

${ }^{11}$ Ibid., p. 175; D 9, 3.

${ }^{12}$ Ibid., p. 177; D 10a, 1.

${ }^{13}$ Walter Benjamin, Kleine Geschichte der Photographie, in Das Kunstwerk im Zeitalter der technischen Reproduzierbarkeit, Frankfurt a. M.: Suhrkamp, 1963, p. 57. „Als erster desinfiziert [Atget] die stickige Atmosphäre, die die konventionelle Porträtphotographie der Verfallsepoche verbreitet hat. Er reinigt diese Atmosphäre, ja bereinigt sie: er leitet die Befreiung des Objekts von der Aura ein, die das unbezweifelbarste Verdienst der jüngsten Photographenschule ist. [...] Er suchte das Verschollene und Verschlagene, und so wenden auch solche Bilder sich gegen den exotischen, prunkenden, romantischen Klang der Stadtnamen; sie saugen die Aura aus der Wirklichkeit wie Wasser aus einem sinkenden Schiff.“ 
sie Riegl umschreibt, und damit der letzte Wink eines entschwundenen Gottes. „Stimmung und Andacht [so schreibt Riegl in seinem Aufsatz] wohnen enge beieinander. Ist doch Andacht nichts anderes als religiöse Stimmung." ${ }^{14}$ Der Zerfall des Stimmungshaften, der Zerfall der Aura, ist der tragische und notwendige Vorbote einer kommenden Katastrophe und der Wunsch der Zerstörung der unauthentischen Aura, ist bei Benjamin von dem Wunsch bestimmt den Historismus und der mit ihm, in seiner Dekadenz, auftauchenden und überbordenden, „träumerischen Zeit des schlechten Geschmacks" ${ }^{\text {"15 }}$ zu überwinden, in der alles zur Staffage wird und in der sich der Nihilismus in der

bürgerlichen Gemütlichkeit [kundtut]: eine Stimmung [, so Benjamin,] die sich im Haschischrausche zu satanischem Genügen, satanischem Wissen, satanischem Ruhen verdichtet. [In diesen Innenräumen] leben [, so Benjamin weiter,] war ein dichtes sich eingewebt, sich eingesponnen haben in ein Spinnennetz, in dem das Weltgeschehen verstreut, wie ausgesogene Insektenleiber herumhängt. ${ }^{16}$

Es ist die Zeit des Unauthentischen von dem auch Adorno sprechen wird und das auch ein wichtiger Angriffspunkt in Heidegger's Technikund Gesellschaftskritik ist. Diese Verfallsepoche, ist durch ein paradoxes Verhältnis zur Natur gekennzeichnet, das sich durch das Bedürfnis bemerkbar macht, das Organische im Anorganischen zu retten und das damit die Entfremdung von der Natur durch ein Aufladen der Kultur mit dem Natürlichen kompensiert. Legte, wie Benjamin schreibt, schon der „Bürger, der mit Louis-Philippe heraufkam [, zwischen 1830 und 1848,] Wert darauf, sich die Natur zum Interieur zu machen" ${ }^{\text {"17 }}$, so ist der ihm folgende Jugendstil nur ein weiterer Schritt in eine träumerische, ornamentale und überbordende Naturaneignung. ,Im Jugendstil [, so notiert Benjamin weiter,] beginnt das Bürgertum mit den Bedingungen [...] seiner Herrschaft über die Natur sich auseinanderzusetzen.“18 „Der Jugendstil forciert das Auratische" 19 , konstatiert Benjamin und er erkennt zugleich, dass die Gier nach der Jugend und dem Organischen im Grunde nur eine Flucht vor dem kommenden Ende ist: „Das Bürgertum fühlt, dass es nicht mehr lange zu

\footnotetext{
${ }^{14}$ Alois Riegl, Stimmung als Inhalt moderner Kunst, p. 55.

${ }_{15}$ Walter Benjamin, Das Passagen-Werk, p. 282; I 1, 6.

${ }^{16}$ Ibid., p. 286; I 2, 6.

${ }^{17}$ Ibid., p. 291, I 4, 1.

${ }^{18}$ Ibid., p. 694, S 9, 4.

${ }^{19}$ Ibid., p. 692, S 8, 8, Cf. Walter Benjamin, Zentralpark, GW, Band I, 2. p. 674.
} 
leben hat; desto mehr will es sich jung [, schreibt Benjamin]. Es spiegelt sich so ein längeres Leben vor oder zumindest einen Tod in Schönheit." ${ }^{\text {20 }}$ An dem Phänomen eines Verfalls der Aura, der Stimmung, der Ruhe und der Ferne, hat aber nach Benjamins Urteil „die Photographie [...] entscheidend teil" ${ }^{\text {"21 }}$, aber Benjamin unterscheidet in seiner Geschichte der Photographie $^{22}$ ganz deutlich zwischen den Anfängen der Fotografien eines Hill und Dautheney, die das Auratische noch im Hauchkreis ${ }^{23}$ der

${ }^{20}$ Ibid., p. 695, S 9a, 4, „Das Verhältnis des Jugenstilinterieurs zu dem ihm vorangehenden beseht darin, daß der Bourgeois sein Alibi in der Geschichte mit dem noch entlegeneren in der Naturgeschichte (besonders dem Pflanzenreiche) vertuscht. “ Cf. Das Passagen-Werk, p. 298 ; I 7,5.

${ }^{21}$ Walter Benjamin, Über einige Motive bei Baudelaire, GW Bd. I,2. p. 644. / Ibid., pp. 646-647. „Der Angesehene oder angesehen sich Glaubende schlägt den Blick auf. Die Aura einer Erscheinung erfahren, heisst, sie mit dem Vermögen belehnen, den Blick aufzuschlagen.“ / Ibid., p. 650. Der sichere Blick enträt der träumerischen Verlorenheit an die Ferne. Er kann dahin kommen, etwas wie Lust an ihrer Entwürdigung zu empfinden. In diesem Sinne dürfen die folgenden merkwürdigen Sätze zu lesen sein. Im „Salon von 1859“ lässt Baudelaire die Landschaftsbilder Revue passieren, um mit dem Eingeständnis zu schliessen: „Ich wünsche mir die Dioramen zurück, deren ungeheure und grobschlächtige Magie mit eine nützliche Illusion aufzwingt. Ich sehe mir lieber ein paar Theaterhintergründe an, auf denen ich kunstfertig, in tragischer Konzision, meine liebsten Träume behandelt finde. Diese Dinge, die so ganz falsch sind, sind eben darum der Wahrheit unendlich viel näher; dagegen sind unsere meisten Landschafter Lügner, gerade weil sie zu lügen verabsäumen." Man möchte auf die ,nützliche Illusion“ weniger Wert legen als auf die ,tragische Konzision'. Baudelaire dringt auf den Zauber der Ferne; er misst das Landschaftsbild geradezu am Maßstab von Malereien in Jahrmarktbuden.“

${ }^{22}$ Walter Benjamin, Kleine Geschichte der Photographie, p. 50. „Strukturbeschaffenheiten, Zellgewebe, mit denen Technik, Medizin zu rechnen pflegen - all dies ist der Kamera ursprünglich verwandter als die stimmungsvolle Landschaft oder das seelenvolle Porträt.“/ Ibid., p. 53. „Man muss im übrigen, um sich die gewaltige Wirkung der Daguerreotypie im Zeitalter ihrer Entdeckungen ganz gegenwärtig zu machen, bedenken, $\mathrm{da} ß$ die Pleinairmalerei damals den vorgeschrittensten unter den Malern ganz neue Perspektiven zu entdecken begonnen hatte. [...] Im Augenblick, da es Daguerre geglückt war, die Bilder der camera obscura zu fixieren, waren die Maler an diesem Punkte vom Techniker verabschiedet worden. Das eigentliche Opfer der Photographie aber wurde nicht die Landschaftsmalerei, sondern die Porträtminiatur.“

${ }^{23}$ Ibid., p. 55. „Soviel vom technischen Bedingtsein der auratischen Erscheinung. [...] Es ist dieser Hauchkreis, der schön und sinnvoll bisweilen durch die nunmehr altmodische ovale Form des Bildausschnitts umschrieben wird. [...] Diese Bilder sind in den Räumen entstanden, in denen jedem Kunden im Photographen vorab ein Techniker nach der neuesten Schule entgegentrat, dem Photographen aber in jedem Kunden der Angehörige einer im Aufstieg befindlichen Klasse mit einer Aura, die bis in die Falten des Bürgerrocks oder der Lavallière sich eingenistet hatte. Denn das bloße Erzeugnis einer primitiven Kamera ist jene Aura nicht. Vielmehr entsprechen sich in der Frühzeit Objekt und Technik genau so scharf, wie sie in der anschließenden Verfallsperiode auseinandertreten.“ 
Fotografien festhielten und es in gewisser Weise noch stärker als die Malerei bedeuten konnten - und der nachfolgenden Verflachung der Fotografie. Während die Aura in den ersten Fotografien, bedingt durch die noch unausgefeilte Technik, wie von selbst hervorscheint, ist die späte Generation der Fotografen damit beschäftigt, so schreibt Benjamin, ,diese Aura durch alle Künste der Retusche, insbesondere jedoch durch sogenannte Gummidrucke vorzutäuschen. " ${ }^{24}$ Die authentische Aura wird im Verlauf der Verfeinerung der Technik, aber vor allem im Verlauf der Entfremdung der industriellen Gesellschaft von der Natur und der damit erfolgenden „Verblassung des Naturschönen“ ${ }^{25}$, von einer scheinhaften Aura verdrängt. Aus einer fortschreitenden Trennung von der Natur und der Unterdrückung des Natürlichen im Kapitalismus, entsteigt schließlich ein völlig unauthentisches Landschaftsgefühl, das sich im pittoresken, touristischen und nationalistischen Landschaftsbedürfnis entfaltet. Ist die Landschaftsmalerei eines van Gogh oder Cézanne noch der Hort in dem sich das Stimmungsvolle bis zur Jahrhundertwende authentisch zu erhalten trachtet, dann ist, wie auch Benjamin zeigt, die industrielle Fotografie, und ihr potenziertes Derivat, die Kinematografie, sowie das auf die Reproduktion getrimmte kapitalistische System, der Hauptgrund des Zerfalls der Stimmung. So wie die Fotografie den Verfall der Aura einleitet, setzt sie auch die Landschaftsmalerei an ihre äußersten Grenzen. Die Fotografie, ,appelliert an das Objektiv [und die] Malerei ihrerseits beginnt die Farbe zu betonen." ${ }^{26}$ Sie, so Benjamin, „muss sich gefallen lassen am Maßstab der Photographie gemessen zu werden. " ${ }^{\text {27 }}$ Der Zerfall der Aura und der Stimmung ist ein Kollateralschaden der telematischen Gesellschaftsentwicklung, dass heißt des Verlusts von Ruhe und Ferne im Zeitalter der technischen und und insbesondere telematischen Errungenschaften, wie die der Fotografie, der Telegrafie, der Kinematografie, der Telekommunikation und der Television. Ruhe und Fernsicht, wie sie Riegl als grundlegend für die Erfahrung der Stimmung beschreibt, werden durch die beschleunigte Bewegung und die Nahsicht hinweggefegt. Und hier lässt sich auch deutlich eine Verwandtschaft zwischen Benjamin und Heidegger erkennen, zumindest im Hinblick auf die Analyse der telematischen Gesellschaft, der Moderne, die sich durch die Abstandslosigkeit auszeichnet und die dabei weder Ruhe noch Ferne bewahrt.

\footnotetext{
${ }^{24}$ Ibid., p. 56.

25 Theodor W. Adorno, Ästhetische Theorie, 13. Aufl., Frankfurt a. M.: Suhrkamp, 1995, p. 112, pp. 97-134.

${ }^{26}$ Walter Benjamin, Das Passagen-Werk, p. 833; Y 5, 3.

${ }^{27}$ Ibid., p. 838; Y 7, 5. Cf., Das Passagen-Werk, pp. 675-676; S 1, 4.
} 
Das 19. Jahrhundert, von dem sich das Werk Simmels nährt und das Benjamin in seiner Passagen-Arbeit auferstehen lassen will, ist der heiße Sommernachmittag, das schwüle Klima einer dekadenten bürgerlichen Kultur, Opfer der Langeweile und der ewigen Wiederkehr. Es wäre auch möglich, diesen leisen Übergang oder diesen Stimmungswechsel anhand der Begriffe, Gefühl, Stimmung, Rausch und Apathie, zu beschreiben, und die rauschhafte Epoche als jene zu erkennen, in der die europäischen Nationen durch den Blutrausch des 1. und des 2. Weltkriegs eintreten und während der Benjamin in seinen Haschischnotizen von 1928 notiert: „[Ernst] Bloch wollte leise mein Knie berühren. Die Berührung wird mir schon lange ehe sie mich erreicht hat, spürbar, ich empfinde sie als höchst unangenehme Verletzung meiner Aura. ${ }^{\text {" }}$ "Seit dieser ersten Beschreibung der Aura, in den Haschischnotizen, steht sie bei Benjamin immer in einer gewissen Verbindung mit dem Landschaftsgefühl, denn, so schreibt Benjamin in einer anderen Haschischnotiz von 1930:

Vielmehr ist das Auszeichnende der echten Aura: das Ornament, eine ornamentale Umzirkung in der das Ding oder das Wesen fest wie in einem Futteral eingesenkt liegt. Nichts gibt vielleicht von der echten Aura einen so richtigen Begriff wie die späten Bilder van Gogh's, wo an allen Dingen - so könnte man diese Bilder beschreiben - die Aura mit gemalt ist. ${ }^{29}$

Was van Gogh kurz vor seinem Selbstmordversuch auf einem Kornfeld von Auvers malt, sind Landschaften, die bereits in das Äußerste gehen, die noch die Stimmung oder Aura zu halten vermögen, deren Zersplitterung auf den Schlachtfeldern des 1. Weltkriegs aber endgültig besiegelt ist. Wer die Kriegslandschaften von Otto Dix im Vergleich zu dem Feld mit Korngarben von van Gogh von Juli 1890 setzt, erkennt, dass die Stimmung nicht nur eine andere ist, sondern dass die Stimmung oder besser doch die Aura, nicht mehr an der Landschaft erfahrbar sind, dass mit dem 20. Jahrhundert die Stimmungslosigkeit im Sinne der Sprachlosigkeit Einzug gehalten hat. Die Frage nach dem was (jetzt) noch Kunst sei, kann nicht mehr an der Landschaftsmalerei abgearbeitet werden. Mit der technischen Reproduktion des Todes in Verdun und in Auschwitz, ist die Stimmung und Stimme des 18. und 19. Jahrhunderts zerbrochen. ${ }^{30} \mathrm{Ab}$ diesem Zeitpunkt

${ }^{28}$ Walter Benjamin, Über Haschisch, Frankfurt a. M.: Suhrkamp Verlag, 1973, p. 73.

${ }^{29}$ Ibid., p. 107.

${ }^{30}$ Bernhard Mendes Bürgi (et al.), Zwischen Erde und Himmel - van Goghs Landschaften. Eine Einführung, in Vincent van Gogh. Zwischen Erde und Himmel. Die Landschaften, Basel: Kunstmuseum Basel, 2009, p. 29. "Im 19. Jahrhundert wurde die Landschaftsmalerei zu einem Experimentierfeld der sich formierenden Moderne: Gerade 
dringt der Begriff der Stimmung aus der Malerei in die Philosophie ein und entfaltet sich, insbesondere in der Existenzialphilosophie, als ein Axiom der Erfahrbarkeit von Welt. Die Stimmung, die Erfahrung des Ganzen, der Ruhe und der Ferne, ist scheinbar unmöglich geworden, doch das metaphysische Bedürfnis der Philosophie sucht weiterhin nach ihr. Heidegger entfaltet seine Philosophie der Stimmungen zum selben Zeitpunkt an dem Benjamin in seinem Kunstwerkaufsatz über den Zerfall der Aura spricht. In seiner Vorlesung Die Grundbegriffe der Metaphysik von 1929/30 erkennt Heidegger die Grundstimmung seiner Gegenwart in der Langeweile, die nicht mehr der traumhafte opiumgeschwängerte und ornamentale Ennui des 19. Jahrhunderts ist. Die Langeweile von der Heidegger schreibt, ist bereits eine Form der Ungestimmtheit, der Stimmungslosigkeit, die Erfahrung der Leere, die im Zeitvertreib, im Konsum, ihre Erfüllung sucht, die bereits ein Ergebnis der Moderne ist und die nach dem Zerfall der Aura deren Platz einnimmt. Diese Langeweile, die sich im Zeitalter der technischen Reproduzierbarkeit in die Seele der Masse ergießt, ist die Vorstufe zur Apathie, die selbst wiederum die Endstufe einer allgemeinen Stimmungs-Ökonomie ist. Die Apathie, die pathologische und nicht die auf einer umfassenden Ethik beruhende antike $\dot{\alpha} \pi \alpha ́ \theta \varepsilon 1 \alpha$, ist die völlige Abwesenheit von Stimmung und Bestimmung und sie ist eine der Masken der Orientierungslosigkeit. Sie bildet, so schreibt Carus in seinen Briefen zur Landschaftsmalerei, das letzte Stadium

in jeder individuellen Lebensform [in der] auf vier Stadien hingewiesen werden [kann], welche als Entwicklung und vollendete Darstellung, Verwelkung und völlige Zerstörung sich unterscheiden lassen. [...] Das Gefühl des Aufstrebens, der Ermuthigung, der Entwickelung, das Gefühl wahrer innerer Klarheit und Ruhe, das Gefühl des Hinwelkens, der Schwermuth und die Fühllosigkeit, Apathie, sind auch hier die vier Stadien, auf welche, als auf die ursprünglichen Grundtöne, das Gemütsleben mit all seiner unendlichen Mannigfaltigkeit sich zurückführen lässt[.] ${ }^{31}$

weil sie nach akademischen Regeln als Gattung gering bewertet wurde, bot sie den Malern neue Möglichkeiten. Delacroix, Courbet und die Impressionisten, sie alle gelangten über Landschafts- und Naturdarstellungen zu innovativen Ausdrucksmöglichkeiten. Die gemalte Natur wurde zum Spiegel der Gefühle, Bedürfnisse und Sehnsüchte einer Gesellschaft, die im Begriff war, von der Industrialisierung grundlegend verändert zu werden. Die Malerei, seit einiger Zeit in Konkurrenz zur Fotografie, wurde sich hier der Möglichkeiten und Grenzen des eigenen Mediums bewusst. Es ist bezeichnend, dass der Weg von der traditionellen Gegenstandsbezeichung hin zur Autonomie der bildnerischen Mittel sowohl bei Wassily Kandinsky als auch bei Piet Mondrian Anfang des 20. Jahrhunderts über eine Auseinandersetzung mit der Landschaftsmalerei führte.”

${ }^{31}$ Carl Gustav Carus, Neun Briefe über Landschaftsmalerei geschrieben in den Jahren 1815-1824, pp. 45-47. 
Jede Epoche entspringt aus einem Wechsel der Töne, hat ihre eigenen Stimmungen und die Stimmung der heutigen Epoche ist die Stimmung der Apathie. Der Mensch spürt sich immer weniger und noch weniger spürt er das, was ihn umgibt. Das, was heute und in naher Zukunft die Menschheit verstimmt, ist die Apathie und eine daraus sich entrollende Ignoranz - die willentliche Unfreiheit sich seines eigenen Verstandes zu bedienen. Die Apathie ist keine einfache Disposition, sondern sehr komplex. Sie ist sichtbar in vielen Formen. Das Schwerwiegende jedoch ist ihre Gewalt. Und schwerwiegend ist die Atmosphäre in der Hinterstube der Apathie: Die Apathie ist tolerant und korrupt zugleich.

\section{BIBLIOGRAPHIE}

Adorno, Theodor W. (1995), Ästhetische Theorie, 13. Aufl., Frankfurt a. M.: Suhrkamp Verlag.

Benjamin, Walter (1973), Über Haschisch, Frankfurt a. M.: Suhrkamp Verlag.

- (1963), Das Kunstwerk im Zeitalter seiner technischen Reproduzierbarkeit, Frankfurt a. M.: Suhrkamp Verlag.

— (1991), Das Passagen-Werk, Frankfurt a. M.: Suhrkamp Verlag.

Busch, Werner (Org.) (1997), Landschaftsmalerei, Berlin: Dietrich Reimer Verlag.

Carus, Carl Gustav (1831), Neun Briefe über die Landschaftsmalerei geschrieben in den Jahren 1815-1824, Leipzig: Verlag Gerhard Fleischer.

Fürnkäs, Josef (2000), Aura, in Benjamin Begriffe, 1. Band, Frankfurt a. Main: Suhrkamp Verlag.

Mendes Bürgi, Bernhard, Nina Zimmer, Walter Feilchenfeldt (2009) Zwischen Erde und Himmel - van Goghs Landschaften. Eine Einführung, in Vincent van Gogh. Zwischen Erde und Himmel. Die Landschaften, Kunstmuseum Basel, pp. 13-29.

Riegl, Alois (1899), Stimmung als Inhalt moderner Kunst, in Die Graphischen Künste, Jahrgang XXII, Heft I, Wien, pp. 47-56. 


\section{ZUSAMMENFASSUNG}

Der vorliegende Text handelt von der Konsolidierung und der Entwicklung der Landschaftsmalerei während des 19. Jahrhunderts und der Katabase einer gewissen Stimmungsökonomie bis in unsere Tage. Der Prozess der Transformation und der Überwindung der Landschafsmalerei spiegelt die Dekadenz einer gesamten Epoche und beeinflusst die Einsetzung der Stimmungen, ebenso wie die Einführung einer neuen philosophischen Disziplin, der Philosophie der Landschaft, in die Philosophie.

Schlagworte: Stimmung - Aura - Apathie - Landschaftsmalerei - Philosophie der Landschaft

\section{RESUMO}

O presente texto discursa sobre a relação entre a consolidação e o desenvolvimento da pintura paisagista e do conceito da paisagem durante o século 19, e a catábase de uma certa economia das disposições até os nossos dias. O processo da transformação e superação da pintura paisagista espelha a decadência de uma inteira época e influenciando a introdução das disposições, como também a iniciação de uma nova disciplina filosófica, a Filosofia da Paisagem, na Filosofia.

Palavras-chave: Disposição (Stimmung) - aura - apatia - pintura paisagista filosofia da Paisagem

\section{ABSTRACT}

The present text deals with the relation between the consolidation and the development of landscape-painting and the concept of landscape during the $19^{\text {th }}$ century and the catabasis of a certain economy of disposition until our days. The process of the transformation and overcoming of landscape-painting reflects the decadence of an entire epoch, influencing the introduction of the dispositions into philosophy, as well the initiation of a new philosophical discipline, the Philosophy of Landscape.

Key-words: disposition (Stimmung) - aura - apathy - Landscape-painting philosophy of Landscape 